\title{
ASOCIACIÓN DE LA CARIES DENTAL CON EL FLUJO Y LA VISCOSIDAD DE LA SALIVA EN ESCOLARES DE BUCARAMANGA, COLOMBIA
}

\author{
${ }^{1}$ Sonia Patricia Suárez Muñoz, ${ }^{1}$ Mónica Tatiana Prieto Roa, ${ }^{1}$ Diana Carolina Otero Quintero, ${ }^{1}$ Walter Alexander Ojeda Olarte, \\ ${ }^{2}$ Sonia Constanza Concha Sánchez \\ ${ }^{1}$ Odontólogo, U. Santo Tomás, Colombia. \\ ${ }^{2}$ Odontóloga U. Santo Tomás, Magister en Epidemiología U. Industrial de Santander, Candidata a Doctor en Salud Pública U. Nacional de Colombia, \\ Docente F. de Odontología U. Santo Tomás, Colombia.
}

Autor responsable de correspondencia: Sonia Constanza Concha Sánchez

Correo electrónico: sococosa@yahoo.com

\begin{abstract}
RESUMEN
Objetivo: determinar la asociación de la caries dental con la viscosidad, el pH y el flujo salival en escolares de Bucaramanga, Colombia. Materiales y métodos: se realizó un estudio observacional analítico de corte transversal en 90 escolares, en los que se recolectaron las características sociodemográficas, dieta, condición de salud, higiene bucal, viscosidad, $\mathrm{pH}$ y flujo salival como variables explicativas y la presencia de caries dental como variable de salida. Para el análisis estadístico univariado se calcularon medidas de resumen según la naturaleza de las variables. Para el bivariado se aplicó test de chi cuadrado y test exacto de Fisher para las variables cualitativas y prueba t de Student o test de rangos de Wilcoxon para las cuantitativas. Para el análisis multivariado se aplicó regresión binomial y se calcularon las Razones de Prevalencia (RP) y sus intervalos de confianza del 95\% (IC 95\%).

Resultados: ser mayor de 8 años [RP 1,4 IC 95\% 1,3 - 1,4], tener un flujo salival anormal [RP 1,3 IC 95\% 1,2 - 1,3] y una viscosidad salival anormal [RP 1,3 IC 95\% 1,2 - 1,3] se asociaron de manera estadísticamente significativa con la caries dental.

Conclusión: el flujo salival disminuido y la viscosidad aumentada de la saliva se asocian con la presencia de caries dental en la población de escolares evaluados. Es importante considerar estos criterios para analizar el riesgo a caries dental. [Suárez SP, Prieto MT, Otero DC, Ojeda WA, Concha SC. Asociación de la caries dental con el flujo y la viscosidad de la saliva en escolares de Bucaramanga, Colombia. Ustasalud 2013; 12: 91 - 100]
\end{abstract}

Palabras clave: Caries dental, Saliva, Viscosidad.

\section{ASSOCIATION BETWEEN DENTAL CARIES WITH FLOW AND VISCOSITY OF SALIVA IN SCHOLARS FROM BUCARAMANGA, COLOMBIA}

\begin{abstract}
Objective: to determine the association between dental caries with the viscosity, the $\mathrm{pH}$ and the saliva flew in scholars from Bucaramanga, Colombia.

Methods: an analytical cross sectional study was carried out with 90 children in which socio-demographic characteristics, diet, health condition, oral hygiene, viscosity, $\mathrm{pH}$ and saliva flow were collected as explanatory variables and the presence of dental caries as dependent one. In the analysis, summary measures were calculated according to the nature of each variable. For univariate statistical analysis summary measures were calculated according to the nature of the variables. For bivariate chi-square test and Fisher exact test for qualitative variables and Student $\mathrm{t}$ test or Wilcoxon rank test for quantitative variables was applied. For binomial regression multivariate analysis was applied and Prevalence Ratios (PR) and confidence intervals of 95\% (CI 95\%) were calculated.

Results: it was found that being over eight years [PR 1.4 CI 95\% 1.3 - 1.4], having an abnormal salivary flow [PR 1.3 CI 95\% 1.2 - 1.3 ] and abnormal salivary viscosity [PR 1.3 CI 95\% 1.2 - 1.3] were statistically significantly associated with dental caries.

Conclusion: decreased salivary flow and increased viscosity of saliva were associated with the presence of dental caries in the school population evaluated. It is important to consider these criteria to analyze the risk to dental caries.
\end{abstract}

Key words: Dental caries, Saliva, Viscosity.

Recibido para publicación: junio 18 de 2013. Aceptado para publicación diciembre 12 de 2013. 


\section{INTRODUCCIÓN}

La caries dental es la enfermedad bucal que afecta a más personas en todo el mundo, en especial a aquellas que pertenecen a los grupos más vulnerables y en condiciones de desventaja. En muchos países se han realizado actividades preventivas con cierto grado de eficacia. Sin embargo, esta entidad sigue apareciendo y causando gran daño en niños y adultos. Investigaciones en Colombia muestran cómo la caries dental afecta hasta el $90 \%$ de los escolares. ${ }^{1}$

La caries de la infancia temprana se ha catalogado como la enfermedad crónica más común en la niñez, es cinco veces más habitual que el asma y gran parte de los niños afectados no reciben tratamiento odontológico o solo en los estadios avanzados de la enfermedad. ${ }^{2}$ Según el último Estudio Nacional de Salud Bucal (ENSAB III), la prevalencia de caries dental en la dentición primaria es del 54,8\% a los 5 años y de $63,8 \%$ a los 7 años. ${ }^{3}$

Esta enfermedad se considera multifactorial, relacionada con diversos factores sociales (condición socioeconómica, nivel de escolaridad y vinculación al sistema de salud, entre otros) y biológicos (condiciones propias del individuo ya sean bucodentales y microbiológicas), trayendo consigo el desequilibrio en el ambiente bucal normal, favoreciéndose así la proliferación de microorganismos cariogénicos e inhibiendo los efectos protectores del sistema inmune y de los mecanismos de remineralización que usualmente están presentes en la saliva. ${ }^{4}$

Los factores económicos y los relacionados con el ambiente cultural pueden alterar la ingesta de una dieta protectora, la adopción de medidas de autocuidado, el uso de elementos preventivos influyendo en el desarrollo de lesiones cariosas cavitacionales. Sin embargo, la mayoría de estos aspectos pueden llegar a modificarse, por lo cual, la caries dental puede catalogarse como una patología controlable y en la que puede evitarse su evolución a estadios más avanzados e irreversibles. Todo esto ha motivado a los clínicos y a los investigadores a buscar indicadores que permitan predecir su aparición. ${ }^{4,5}$

La saliva es uno de los componentes que mantiene un ambiente bucal adecuado puesto que lubrica los tejidos finos, mantiene un $\mathrm{pH}$ neutro que promueve la remineralización del diente a través de los minerales disponibles y ejerce una acción antimicrobiana mediante las inmunoglobulinas presentes en este líquido. Además, cumple un papel crítico en la prevención del proceso de la caries dental, al proporcionar calcio, fosfato y proteínas que mantienen la sobresaturación del calcio en la placa, proteínas y lípidos que forman una película protectora sobre el diente y condicionan el $\mathrm{pH}$ y la viscosidad de la saliva. $^{6}$

Es por esto que el conocimiento de las características de la saliva, como son el flujo, el pH y la viscosidad pueden ser importantes para determinar el estado de salud bucal, su influencia sobre el medio ambiente oral y su efecto protector sobre la caries dental en las diferentes edades.?

En la actualidad hay pocos estudios que analicen las variaciones de flujo salival en niños, no se evidencian además, parámetros que permitan determinar en ellos los niveles de flujo salival normal por lo que es dificil identificar el impacto de este factor en el riesgo sobre la caries dental. Una tasa de flujo salival adecuada es esencial para que la salud bucal se mantenga, pero este equilibrio puede interrumpirse al alterarse el balance entre el huésped y los microorganismos, al dar lugar al crecimiento excesivo de bacterias. ${ }^{5}$ Es por esto que la evaluación del flujo salival es una herramienta esencial que contribuye a estimar el riesgo de caries dental en una persona.

El promedio del flujo salival en adultos oscila entre 0,30 y $0,5 \mathrm{~mL} / \mathrm{min}$ en saliva no estimulada y entre 2,0 y $3,0 \mathrm{~mL} / \mathrm{min}$ en saliva estimulada, ${ }^{8}$ lo cual ayuda a identificar un potencial de riesgo de caries dental asociado a la disminución de estos valores en los adultos. No obstante, no es claro si estos mismos parámetros podrían emplearse para evaluar el nivel de riesgo en la población escolar. ${ }^{9}$ Adicional al flujo, la medición del pH y la viscosidad de la saliva son otros aspectos que pueden llegar a condicionar el desarrollo de lesiones cariosas en los niños. Sin embargo, la literatura al respecto es limitada.

Al establecer una medición de flujo salival estimulado, la viscosidad y el $\mathrm{pH}$ salival en infantes, se busca generar evidencia que oriente acciones dirigidas a promover la salud y prevenir las enfermedades bucodentales. Mostrando así la importancia que tiene la saliva como factor protector, su injerencia sobre el medio ambiente bucal y su potencial efecto sobre otros factores que pueden aumentar el riesgo de caries dental.

Por tal motivo, el objetivo del presente trabajo fue determinar la asociación de la caries dental con la viscosidad, el $\mathrm{pH}$ y el flujo salival estimulado en niños entre los 6 y 12 años de edad que se encontraban vinculados con dos instituciones educativas de la ciudad de Bucaramanga.

\section{MATERIALES Y MÉTODOS}

Se realizó un estudio observacional analítico de corte transversal. ${ }^{10} \mathrm{El}$ universo estuvo compuesto por niños de ambos géneros entre los 6 y 12 años, estudiantes de dos instituciones educativas públicas 
del área metropolitana de Bucaramanga. Se incluyeron los niños matriculados de primero a quinto de primaria de las instituciones seleccionadas, cuyos padres y ellos mismos aceptaran vincularse a la investigación. Se excluyeron los niños con patologías en glándulas salivales y aquellos que manifestaron su deseo de no participar en el estudio.

El tamaño de muestra se definió a partir de los siguientes criterios: un nivel de confianza del 99\%, poder del $80 \%$, relación expuesto: no expuesto $1 \mathrm{a}$ 1 , frecuencia de caries dental en personas con flujo salival disminuido de $45,8 \%$, y con flujo salival normal del 8,4\%. ${ }^{11} \mathrm{Al}$ considerar una atrición del 20\% se obtuvo un tamaño de 90 escolares mediante la rutina Stat-cal del paquete estadístico EPI-INFO 6.04 para estudios de corte transversal. ${ }^{12}$

Las variable de salida fue la presencia de caries dental. Para su valoración se tuvo en cuenta el índice de dientes Cariados, Obturados y Perdidos (COP) y también se evalúo la presencia de mancha blanca. Para el COP se considero analizarse en forma conjunta (COP-d) así como cada uno de sus componentes mediante la determinación del número de dientes Cariados (C), Obturados (O) y Perdidos (P). Por lo tanto se catalogaron como variables cuantitativas, discretas, medidas en escala de razón. ${ }^{3}$ Así mismo, se determinó la presencia de caries dental cuando el índice $\mathrm{COP}$ reportó valores mayores o iguales a uno y ausencia de esta cuando el COP era igual a cero lo que permitió operacionalizar la variable como cualitativa, nominal. La mancha blanca se evalúo según el número de dientes que registraban esta condición.

Entre las variables explicatorias se encontraban las características sociodemográficas (edad, género, estrato socioeconómico de la familia, nivel educativo y estado laboral de la madre). Además, se registraron las siguientes:

- Dieta: para recolectar esta variable se utilizó el cuestionario sobre consumo de alimentos propuesta por Lipari y Andrade y citada por Vaisman y Martínez, que cuantifica el potencial cariogénico de la dieta (cuantitativa, continua en escala de razón) y también categoriza el puntaje de riesgo de la dieta al catalogar como bajo cuando los valores oscilan entre 10 y 33 puntos, moderado cuando se encuentran entre 34 y 79 y de alto al obtener puntajes entre 80 y 144 . Por lo tanto, también fue catalogada como una variable cualitativa medida en escala ordinal. ${ }^{13}$

- Estado general de salud: definida como la presencia de alguna patología. Se clasificó como cualitativa, nominal. Su nivel operativo fue presencia 0 ausencia de alteraciones, cardiovasculares, gas- trointestinales, nerviosas, respiratorias, hepáticas, siquiátricas, endocrinas, músculo-esqueléticas, alérgicas, de la piel, visuales, auditivas, genitourinarias o cirugías.

- Ingesta de medicamentos: clasificada en cualitativa, nominal. Su nivel operativo es consume o no consume. Si la respuesta era afirmativa se le indagó el tipo y la cantidad de medicamentos ingeridos.

- Higiene bucal: se determinó al aplicar el índice cuantificado de placa (ICP) según lo establecido por Pieschacón y Camargo. Esta variable se recolectó en forma cuantitativa y se categorizó como buena $(0-0,9)$ regular $(1,0-1,9)$ o mala $(2,0-3,0) .{ }^{14}$

- Flujo salival: se evaluó mediante el test de flujo salival estimulado de acuerdo con los procedimientos establecidos por Axelsson y Seif y retomado por Ojeda y colaboradores. ${ }^{15}$ Se clasificó como cuantitativa, continua, de razón. Su nivel operativo fue mililitros de saliva estimulada, recolectada en un minuto. Posteriormente, se categorizó como normal cuando el flujo salival fue mayor a $0,7 \mathrm{~mL} / \mathrm{min}$ y anormal cuando registró valores inferiores definidos según los criterios establecidos para el Cariogram. ${ }^{16}$

- pH salival: definida como el grado de acidez o basicidad de la saliva. Se clasificó como cualitativa, ordinal. Su nivel operativo fue ácido (valores inferiores a 7), básico (valores superiores a 7) o neutro (valores iguales a 7). También, se clasificó de acuerdo con lo establecido por la tira de papel tornasol y definido por la casa fabricante.

Los procedimientos se iniciaron con la construcción de la fase conceptual, metodológica y la elaboración del respectivo instrumento. Luego se efectúo la estandarización, se evaluaron los procesos y los cuestionarios mediante la prueba piloto, momento en el que se identificaron inconsistencias en los procesos y en el formato de recolección de datos; se hicieron los correctivos, se asignaron tareas a cada investigador y se procedió a concertar el desarrollo del trabajo con las instituciones educativas involucradas.

Para dar cumplimiento al objetivo propuesto, un investigador procedió a interrogar a la madre o acudiente y al menor sobre los aspectos previamente definidos y se obtuvo el consentimiento informado del acudiente y el asentimiento del participante.

Para la medición del flujo salival, el pH y la viscosidad un evaluador asignado procedió de la siguiente manera: primero se solicitó al menor que deglutiera la saliva que tenía en su boca y se la enjuagara con agua, luego se le administraron tres miligramos de cera rosada previamente reblandecida. Se le pidió que la masticara durante dos minutos, luego el niño 
depositó la saliva en un frasco milimetrado durante cuatro minutos; a continuación, el examinador cuantificó la cantidad de saliva recolectada y el registro lo dividió entre cuatro. Con esta muestra de saliva se procedió a medir el $\mathrm{pH}$ utilizando papel indicador de $\mathrm{pH}$ 1-14 (Mancherey-Nagel ${ }^{\circledR}$ ) y se comparó con la escala de medición de $\mathrm{pH}$ para determinar el grado de acidez o basicidad de la saliva y el valor obtenido de acuerdo con el color que tomaba el papel y establecido por la guía definida por la casa fabricante.

Para evaluar la viscosidad de la saliva, otro de los investigadores tomó una muestra de saliva y agua mediante dos pipetas y valoró cuál de las dos gotas de saliva o agua se precipitaba más rápido; si la gota de saliva se precipitaba más rápido o igual que la de agua la viscosidad de la saliva se catalogaba como normal y si ocurría más rápido con el agua que con la saliva la viscosidad se definía como anormal.

Posterior a la medición de flujo salival, se evaluó la higiene bucal de los escolares, la información se recolectó siguiendo los parámetros establecidos para el Índice Cuantificado de Placa (ICP), procediendo de la siguiente manera: con el niño sentado frente al evaluador responsable, se aplicó la solución reveladora de placa, que el menor llevó a las superficies de los dientes ayudado con su lengua. Se tomaron los tres dientes superiores y se observaron por vestibular y los tres inferiores que se examinaron por lingual, cada superficie evaluada se dividió imaginariamente en tercios y se marcó una $\mathrm{X}$ en el tercio pigmentado; el resultado de este índice se consolidó al sumar los tercios pigmentados y dividirlos por el total de dientes evaluados.

A continuación un operador efectúo un cepillado supervisado de las superficies dentarias, actividad que realizó con el cepillo y crema dental que se le entregó a cada escolar; luego, evaluó la presencia de caries dental de acuerdo con criterios establecidos para el COP procediendo de la siguiente manera:

Con el niño sentado frente al operador, quien con el espejo y una sonda periodontal WHO y sin ejercer presión retentiva en ningún momento procedió a determinar la existencia de lesiones cariosas, dientes obturados o perdidos por caries dental; posteriormente, se examinó la superficie vestibular de cada uno de los dientes presentes en la boca del menor y en los que se determinó la existencia de mancha blanca y el número de dientes afectados por esta condición, toda la información se recolectó en el formato respectivo.

Luego, se procedió a realizar la codificación, siste- matización por duplicado de la información en archivos en Excel y mediante la rutina Validate del paquete EPI-INFO 6.04, se verificó la calidad de la digitación; se hicieron los correctivos necesarios y se exportó al paquete estadístico STATA 9.0 para su procesamiento y análisis. ${ }^{12,17,18}$

\section{Plan de análisis estadístico}

Para el análisis univariado se calcularon medidas de resumen según la naturaleza de cada variable. Para el análisis bivariado se consideró como variable de salida presencia de caries dental (dicotomizada) la cual se relacionó con las variables explicativas; para ello se aplicaron pruebas estadísticas como $\mathrm{Chi}^{2}$ o Test Exacto de Fisher para variables cualitativas y para las variables cuantitativas se aplicó la prueba t de Student o test de rangos de Wilcoxon según la distribución de los datos. ${ }^{19}$

En el análisis multivariado, se realizó una regresión binomial. Se calcularon las Razones de Prevalencia (RP) con los respectivos intervalos de confianza. ${ }^{20}$ Se consideró un nivel de significancia de $\mathrm{p} \leq 0,05$ para todos los análisis.

\section{Consideraciones éticas}

La presente investigación se acogió a las normas establecidas en la Resolución 008430 del Ministerio de Salud, ${ }^{21}$ de la Republica de Colombia y a lo establecido en la Declaración de Helsinki. Se garantizaron los principios de beneficencia, equidad, privacidad, confidencialidad y veracidad en el manejo de la información recolectada, para dar cumplimiento al principio da autonomía se obtuvo el consentimiento informado del acudiente y el asentimiento del niño.

\section{RESULTADOS}

\section{Análisis univariado}

\section{Variables sociodemográficas y las relacionadas con salud general}

Se analizaron noventa escolares con un promedio de edad de $8,8 \pm 1,8$ años, el 51,1\% (46) eran hombres y el $86,7 \%$ (78) estaba vinculado a una fundación que acoge a población desplazada. El 54,4\% (49) reportó ser del estrato socioeconómico 1, el 45,6\% (41) de los escolares estaba en segundo grado de primaria, el 76,7\% (68) de las madres de los niños cursaron solo nivel educativo primario, el 66,7\% (60) de las madres trabajaba y el $47,8 \%$ (43) convivía con sus dos progenitores. Adicionalmente, se evidenció la presencia de caries dental en el 64,4\% (58) de los escolares (Tabla 1).

\section{Historia de caries dental}


Al estimar la historia de caries dental, el promedio del COP fue 3,4 $\pm 2,7$ dientes y el promedio de dientes cariados fue $1,5 \pm 1,8$ y de obturados de $1,4 \pm$ 1,9 dientes. Se resalta el hecho que el promedio más alto se registró para las lesiones de mancha blanca $(1,8 \pm 1,3)$ (Tabla 2$)$.

\section{Factores de riesgo asociados con caries dental}

El 90\% (81) de los escolares consumía una dieta que se catalogó de riesgo moderado para la caries dental, el 88,9\% (80) registró una higiene bucal regular, el $75,6 \%$ (68) un flujo salival anormal, 41,4\% (37) viscosidad salival anormal y el $36,7 \%$ (33) un pH salival neutro (Tablas 3 y 4).

Tabla 1. Análisis de las variables sociodemográficas

descritas en forma global y según presencia de caries dental

\begin{tabular}{|c|c|c|c|}
\hline Variable & $\begin{array}{c}\text { Global } \\
\text { n (\%) }\end{array}$ & $\begin{array}{c}\text { Presencia de caries dental } \\
\text { n (\%) }\end{array}$ & $\mathbf{P}^{*}$ \\
\hline Edad categorizada (años) & & & $<0,001 \dagger$ \\
\hline $6-8$ & $49(54,4)$ & $23(46,9)$ & \\
\hline $9-12$ & $41(45,6)$ & $35(85,4)$ & \\
\hline Género & & & $0,770^{\dagger}$ \\
\hline Hombre & $46(51,1)$ & $29(63,0)$ & \\
\hline Mujer & $44(48,9)$ & $29(65,9)$ & \\
\hline Institución & & & $0,004^{*} \dagger$ \\
\hline Número 1 & $12(13,3)$ & $3(25,0)$ & \\
\hline Número 2 & $78(86,7)$ & $55(70,6)$ & \\
\hline Estrato socioeconómico & & & 0,106 \\
\hline Uno & $49(54,4)$ & $36(73,5)$ & \\
\hline Dos & $30(33,3)$ & $15(50,0)$ & \\
\hline Tres & $11(12,2)$ & $7(63,6)$ & \\
\hline Curso (grado) & & & $<0,001 \dagger$ \\
\hline Segundo & $41(45,6)$ & $17(41,5)$ & \\
\hline Tercero & $28(31,1)$ & $21(75,0)$ & \\
\hline Cuarto & $21(23,8)$ & $20(95,2)$ & \\
\hline Nivel educativo madre & & & 0,521 \\
\hline Ninguno & $1(1,1)$ & $1(100)$ & \\
\hline Primaria & $68(76,7)$ & $42(60,9)$ & \\
\hline Secundaria & $17(20,0)$ & $14(77,8)$ & \\
\hline Técnico & $2(2,2)$ & $1(50,0)$ & \\
\hline Murió & $2(2,2)$ & $2(100)$ & \\
\hline Madre trabajadora & & & 0,831 \\
\hline $\mathrm{Si}$ & $60(66,7)$ & $38(69,3)$ & \\
\hline No & $28(31,1)$ & $18(64,3)$ & \\
\hline Murió & $2(2,2)$ & $2(100)$ & \\
\hline Convivencia padres & & & 1,000 \\
\hline Con los dos & $43(47,8)$ & $27(62,3)$ & \\
\hline Sólo con uno & $43(47,8)$ & $28(65,1)$ & \\
\hline Con ninguno & $4(4,4)$ & $3(75,0)$ & \\
\hline Compromiso de salud general & $19(21,1)$ & $15(78,9)$ & 0.181 \\
\hline Sometido a medicación & $8(8,9)$ & $6(75,0)$ & 0.707 \\
\hline
\end{tabular}

${ }^{*}$ Test de $\mathrm{Chi}^{2}{ }^{* *}$ Test Exacto de Fisher † Significancia estadística 
Tabla 2. Descripción de la historia de caries dental analizada como COP y según el componente de cariados, obturados y perdidos

\begin{tabular}{|c|c|c|}
\hline Variable & $\bar{X} \pm$ D.E. & Rango \\
\hline $\mathrm{COP}$ & $3,4 \pm 2,7$ & $0-11$ \\
\hline Cariados & $1,5 \pm 1,8$ & $0-6$ \\
\hline Obturados & $1,4 \pm 1,9$ & $0-7$ \\
\hline Perdidos & $0,4 \pm 1,0$ & $0-7$ \\
\hline Mancha blanca & $1,8 \pm 1,3$ & $0-4$ \\
\hline
\end{tabular}

$\overline{\bar{X}} \pm$ D.E.: Promedio \pm Desviación estándar

Tabla 3. Variables cualitativas relacionadas con el flujo salival y con factores de riesgo a caries dental descritas en forma global y según la presencia de caries dental.

\begin{tabular}{lccc}
\hline \multicolumn{1}{c}{ Variable } & $\begin{array}{c}\text { Global } \\
\mathbf{n}(\%)\end{array}$ & $\begin{array}{c}\text { Presencia de caries dental } \\
\mathbf{n}(\%)\end{array}$ & $\mathbf{P}^{*}$ \\
\hline Dieta de riesgo & & & 0,482 \\
Bajo & $9(10,0)$ & $7(77,8)$ & \\
Moderado & $81(90,0)$ & $51(63,0)$ & 0.485 \\
Higiene bucal & & & \\
Mala & $10(11,1)$ & $8(80,0)$ & \\
Regular & $80(88,9)$ & $50(62,5)$ & 0,927 \\
Flujo salival anormal & $68(75,6)$ & $44(64,7)$ & 0,106 \\
pH & & & \\
Ácido & $26(28,9)$ & $21(88,9)$ & \\
Básico & $31(34,4)$ & $17(54,8)$ & \\
Neutro & $33(36,7)$ & $20(60,6)$ & $0,021 \dagger$ \\
Viscosidad salival anormal & $37(41,1)$ & $29(78,4)$ & \\
\hline
\end{tabular}

${ }^{*}$ Test de $\mathrm{Chi}^{2}+$ Significancia estadística

Tabla 4. Variables cuantitativas relacionadas con el flujo salival y con factores de riesgo a caries dental descritas en forma global y según su presencia.

\begin{tabular}{|c|c|c|c|}
\hline Variable & $\begin{array}{c}\text { Global } \\
\bar{X} \pm \text { D.E. }\end{array}$ & $\begin{array}{l}\text { Presencia de caries dental } \\
\bar{X} \pm \text { D.E. }\end{array}$ & $\mathbf{P}^{*}$ \\
\hline Dieta & $44,5 \pm 9,5$ & $44,5 \pm 10,2$ & 0,950 \\
\hline Higiene bucal & $1,6 \pm 0,3$ & $1,6 \pm 0.2$ & 0,224 \\
\hline Flujo salival & $0.6 \pm 0,2$ & $0,7 \pm 0,2$ & 0,183 \\
\hline pH salival & $7,1 \pm 0,9$ & $6,9 \pm 1,0$ & $0,048 \dagger$ \\
\hline
\end{tabular}

$\overline{\mathrm{X}} \pm$ D.E.: Promedio \pm Desviación estándar. ${ }^{*}$ Prueba t de Student $\quad+$ Significancia estadística 


\section{Análisis bivariado}

\section{Variables sociodemográficas y las relacionadas con salud general}

Las variables sociodemográficas que se asociaron con la presencia de caries dental fueron la edad $(p<0,001)$, institución $(p=0,004)$ y curso $(p<0,001)$. El promedio de edad de los niños con caries dental fue de $9,4 \pm 1,8$ y la de los niños sin caries fue de $7,8 \pm 1,2$ años. Del total de escolares que provenían de una institución que educa población desplazada el 70,6\% (55) registró caries dental mientras que el total de niños vinculados a una institución pública el 25\% (3) presentó esta entidad (Tabla 1).

\section{Factores de riesgo asociados con la caries dental}

$\mathrm{Al}$ analizar los factores bucales relacionados con la caries dental se observó que el $\mathrm{pH}$ salival evaluado en forma cuantitativa $(p=0,048)$ y una viscosidad anormal de la saliva $(p=0,021)$ se asociaron estadísticamente con la presencia de caries dental. Se observó un promedio de pH salival de 6,9 $\pm 1,0$ en las personas que registraron caries dental, mientras que en las personas que no la presentaron fue de 7,3 $\pm 0,9$. En cuanto a la viscosidad se observó que del total de niños que presentaron viscosidad anormal de la saliva el 78,4\% (29) registraban caries dental y el 21,6\% no (Tabla 3).

\section{Análisis multivariado}

Factores de riesgo asociados a caries dental modelo no ajustado

El análisis binomial no ajustado de la asociación de la caries dental mostró que las variables "estar vinculado a la institución dos" (que acoge a población desplazada) [RP 2,8 IC 95\% 1,0 - 7,6], estar en tercero o cuarto grado de primaria [RP 2,0 IC 95\% 1,4 - 3,0] y presentar una viscosidad salival anormal [RP 1,4 IC $95 \% 1,1-1,9]$ se asociaron en forma estadísticamente significativa con la presencia de caries dental (Tabla 5).

Factores de riesgo asociados a caries dental modelo ajustado

El modelo final ajustado mostró que ser mayor de 8 años [RP 1,4 IC 95\% 1,3 - 1,4], tener un flujo salival anormal [RP 1,3 IC 95\% 1,2 - 1,3] y una viscosidad salival también anormal [RP 1,3 IC 95\% 1,2 - 1,3] se asociaron en forma estadísticamente significativa con la caries dental (Tabla 6).

Tabla 5. Asociación de la caries dental con factores de riesgo modelo no ajustado

\begin{tabular}{|c|c|c|c|}
\hline Variable & $\mathbf{R P}$ & IC $95 \%$ & $\mathbf{P}$ \\
\hline \multicolumn{4}{|l|}{ Edad } \\
\hline Menor o igual a 8 años & 1,0 & - & - \\
\hline Mayor de 8 años & 1,8 & $1,3-2,5$ & $<0,0001 \dagger$ \\
\hline \multicolumn{4}{|l|}{ Institución educativa } \\
\hline Institución 1 & 1,0 & - & - \\
\hline Institución 2 & 2.8 & $1,0-7,6$ & $0,04 \dagger$ \\
\hline \multicolumn{4}{|l|}{ Grado escolar } \\
\hline Segundo grado & 1,0 & - & - \\
\hline Tercer y cuarto grado & 2,0 & $1,4-3,0$ & $0,006 \dagger$ \\
\hline \multicolumn{4}{|l|}{ Flujo salival } \\
\hline Normal & 1,0 & - & - \\
\hline Anormal & 1,0 & $0,7-1,5$ & 0,09 \\
\hline \multicolumn{4}{|l|}{ pH salival } \\
\hline Ácido & 1,0 & - & - \\
\hline Básico / neutro & 1,1 & $1,0-1,2$ & $0,02+$ \\
\hline \multicolumn{4}{|l|}{ Viscosidad salival } \\
\hline Normal & 1,0 & - & - \\
\hline Anormal & 1,4 & $1,1-1,9$ & $0,018 \dagger$ \\
\hline
\end{tabular}

† Significancia estadística 
Tabla 6. Asociación de la caries dental con factores de riesgo (modelo ajustado).

\begin{tabular}{|c|c|c|c|}
\hline Variable & $\mathbf{R P}$ & IC 95\% & $\mathbf{P}$ \\
\hline \multicolumn{4}{|l|}{ Edad } \\
\hline Menor o igual a 8 años & 1,0 & - & - \\
\hline Mayor de 8 años & 1,4 & $1,3-1,4$ & $<0,0001 \dagger$ \\
\hline \multicolumn{4}{|l|}{ Institución educativa } \\
\hline Institución 1 & 1,0 & - & - \\
\hline Institución 2 & 2,2 & $0,8-6,0$ & 0,110 \\
\hline \multicolumn{4}{|l|}{ Grado escolar } \\
\hline Segundo grado & 1,0 & - & - \\
\hline Tercer y cuarto grado & 1.0 & $1,0-1,1$ & $0,006+$ \\
\hline \multicolumn{4}{|l|}{ Flujo salival } \\
\hline Normal & 1,0 & - & - \\
\hline Anormal & 1,3 & $1,2-1,3$ & $<0,0001 \dagger$ \\
\hline \multicolumn{4}{|l|}{ pH salival } \\
\hline Ácido & 1,0 & - & - \\
\hline Básico / neutro & 1,1 & $1,1-1,2$ & $<0,0001 \dagger$ \\
\hline \multicolumn{4}{|l|}{ Viscosidad salival } \\
\hline Normal & 1,0 & - & - \\
\hline Anormal & 1,3 & $1,2-1,3$ & $<0,0001 \dagger$ \\
\hline
\end{tabular}

† Significancia estadística

\section{DISCUSIÓN}

La identificación de los factores asociados a la caries dental, en grupos de riesgo específico, es una labor muy importante que debe implementar la profesión odontológica; todo con miras a generar evidencia que fundamente la planeación y aplicación de los programas promocionales y preventivos coherentes con las necesidades observadas. ${ }^{22}$

Sin embargo, a pesar de su importancia, esta tarea no es fácil. La primera razón que fundamenta esta afirmación se sustenta en el origen multifactorial de la caries dental. Además, muchos de los factores que originan esta entidad, no obstante de haber sido identificados, no son fáciles de evaluar. ${ }^{23}$

El estudio de los microorganismos generadores de la caries dental es importante, pero implica procesos relativamente complejos y quizás costosos. Por otra parte, la evaluación del potencial cariogénico de la dieta es un proceso difícil pues implica no solo recolectarla sino también analizarla de una manera válida. ${ }^{24}$

Las condiciones sociales y económicas son otros factores que pueden incidir de diferente manera para que la presencia y aparición de la caries dental se vea modificada. De ahí la importancia de incluir estos aspectos cuando se pretende establecer relacio- nes de asociación de la caries dental con cualquier aspecto de interés en particular. ${ }^{25}$

El uso de fluoruros, de los elementos de higiene bucal, los rasgos morfológicos del diente y las características de la estructura del esmalte también podrían determinar en gran medida la aparición de la caries dental. ${ }^{22}$ Se hace necesario además, considerar el valor que tiene la saliva como un elemento de diagnóstico que ha readquirido importancia en la actualidad. A partir de la saliva se pueden implementar diagnósticos como el análisis de toxinas, la presencia de drogas y la identificación de factores carcinogénicos, entre otros. Pero también se deben reconsiderar aspectos que pueden ser más sencillos pero que podrían tener gran valor en análisis de factores de riesgo para la caries dental y dentro los que cabría destacar la evaluación del flujo, el pH y la viscosidad salival. ${ }^{26}$

El análisis del flujo, el pH y la viscosidad de la saliva pueden constituirse en factores relevantes. La cantidad de saliva, el grado de acidez y la capacidad amortiguadora, así como, la densidad de la saliva pueden llegar a condicionar su capacidad de remineralización; la evaluación de estos aspectos podría realizarse mediante procedimientos relativamente sencillos. ${ }^{27}$ 
Sin dejar reconocer las potenciales limitaciones de las pruebas salivales empleadas, es importarte resaltar los resultados relacionados con los factores asociados con la caries dental en el presente trabajo. El flujo y la viscosidad de la saliva se constituyeron en los aspectos salivales que mostraron mayor asociación con la caries dental. Los flujos salivales disminuidos y la saliva viscosa parecen tener relación con la caries dental ambos con una RP 1,3 IC 95\% 1,2 - 1,3.

El flujo salival disminuidos y la alta viscosidad pueden tener impacto por diferentes razones, el primero podría ser la reducción en los procesos de aclaramiento salival por lo cual la sacarosa consumida por los escolares permanece por periodos más largos de los deseados en la cavidad bucal e incrementa con ello la caída del pH y como consecuencia la desmineralización del esmalte dental, fenómeno que trae consigo los cambios ultra-estructurales relacionados con el inicio de la caries dental. ${ }^{28}$

Adicionalmente, es probable que una saliva viscosa registre mayor cantidad de proteínas del tipo mucinas, que pueden condicionar el contenido iónico responsable de los procesos de remineralización dental, por lo cual el esmalte vulnerado no tiene la capacidad de recuperarse, superando con ello los ciclos de desmineralización a los de remineralización patrón descrito para el inicio de caries dental que puede evolucionar una lesión cavitacional. ${ }^{26}$

Es importante reconocer que en la saliva también se evidencian los sistemas tampón o buffer y los sistemas inmunológicos de tipo específico o no específico que pueden llegar a modular el proceso de génesis de la caries dental. $\mathrm{El} \mathrm{pH}$ aunque positivamente asociado, solo le aporta un $10 \%$ al modelo de caries dental; sin embargo, las implicaciones clínicas de este criterio podrían verse potenciadas cuando se encuentra un menor que además de tener su pH alterado, también presenta poca saliva y muy viscosa. ${ }^{26}$

$\mathrm{Al}$ evaluar la edad como el aspecto sociodemográfico que permaneció como un factor positivamente asociado con la caries dental es importante señalar que ser mayor de 8 años registró una asociación 1,4 [IC 95\% 1,3 - 1,4] veces mayor con la caries dental al comparar con niños de 8 años o menos. Este resultado coincide con el hallado por Segovia-Villanueva y colaboradores quienes afirmaron que en población preescolar la severidad de la caries aumenta con la edad. ${ }^{29}$ No obstante, Sánchez-Pérez y colaboradores sugirieron que la susceptibilidad a la caries decrece con la edad y que las tendencias al aumento o disminución del ataque carioso depende más de la edad posteruptiva, que de la edad del individuo. ${ }^{30}$
Este rasgo en particular parece ser biológicamente consistente con la evidencia, pues los niños de mayor edad tienden a acumular mayores desafios cariogénicos sobre la superficie del esmalte, reconociendo además, que el índice COP refleja la historia de caries de tal manera que se evidencia como un estigma permanente y que la caries cavitacional que se genera se refleja bien a través de este índice.

En los resultados presentados relacionados con el índice $\mathrm{COP}$ se observó que el criterio que más aportó a este indicador fue el de caries dental (C) que registró un promedio de 1,5 dientes cariados, seguido muy de cerca por el componente obturado (0) con un promedio de 1,4 dientes obturados. Quizás el aspecto más relevante es el análisis del promedio de lesiones de mancha blanca pues fue el mayor (1,8 lesiones blancas) dentro de estos criterios analizados y relacionados con la caries dental.

El valor clínico de este hallazgo sugiere la necesidad de implementar acciones de tipo preventivo dentro de las que cabría considerar el uso de elementos fluorurados, el aumento del uso de goma de mascar libre de sacarosa, el fortalecimiento en las medidas de higiene bucal. Pues el mayor promedio de las lesiones cariosas están estadios que podrían revertirse si se implementan estrategias preventivas consistentes con los hallazgos observados. ${ }^{31}$

Una de las limitaciones del presente trabajo fue la forma como se evalúo la dieta en el grupo más joven de los escolares analizados, pues implicó interrogar a los estudiantes sobre su ingesta en el curso de la semana y esto no es un proceso fácil, que podría verse afectado por un sesgo de memoria. Sin embargo, es importante referir que se intentó controlar, pues el proceso de interrogatorio, de acuerdo con lo establecido por el mismo instrumento, implicaba ayudar al menor a devolverse en el tiempo de manera paulatina empezando por la ingesta más cercana. ${ }^{32}$

A manera de conclusión, se puede afirmar que los hallazgos registrados en este trabajo parecen indicar que el flujo disminuido y la viscosidad aumentada de la saliva se asocian con la caries dental en la población de escolares evaluados. Además, los resultados relacionados con las variables socio-demográficas parecen mostrar que a mayor edad hay mayor posibilidad de presentar caries dental.

Estos resultados orientan la importancia y la necesidad de implementar acciones promocionales y preventivas dirigidas a mejorar las condiciones dentales de los escolares evaluados, sin dejar de reconocer, que dichas acciones podrían también tener efecto sobre otras patologías bucales como son la 
enfermedad periodontal e incluso la maloclusión por los factores de riesgo comunes.

\section{Agradecimientos}

Los investigadores de este trabajo agradecen a la Universidad Santo Tomás de Bucaramanga el apoyo financiero a este proyecto.

\section{BIBLIOGRAFÍA}

1. Escobar G. Valoración del riesgo de caries dental, una herramienta para la atención integral del niño. Rev Fac Odontol Univ Antioq 2006; 18: 68 - 80.

2. Douglass JM, Douglass AB, Silk HJ. A practical guide to infant oral health. Am Fam Physician 2004; 11: 2113 21120.

3. Ministerio de Salud. III Estudio Nacional de Salud bucal (ENSAB III). Bogotá: Ministerio de Salud; 1999. p. 85 - 147.

4. Sánchez-Pérez L, Sáenz ML, Irigoyen CE, Luengas AI, Tomasis GJ. Predicción de caries, indicadores de riesgo en saliva y placa dental en niños sanos. Revista Mexicana de Pediatría 2006; 73: 112 - 118

5. Loyo K, Balda R, González O, Solórzano AL, González M, Actividad cariogénica y su relación con el flujo salival y la capacidad amortiguadora de la saliva. Acta Odontol Venez [en línea] 1999; 37 [fecha de acceso: 12 de agosto de 2010]. URL disponible en: http://www.actaodontologica.com/ediciones/1999/3/actividad_cariogenica_relacion_flujo_salival.asp

6. Feartherstone JD. The science and practice of caries. J Am Dent Assoc 2000; 131: 887 -899.

7. Lázaro CP, Valença AMG, Chiappini CC. Estudo preliminar do potencial cariogênico de preparações doces da merenda escolar através do $\mathrm{pH}$ da saliva. Rev. Nutr. [en línea] 1999 [fecha de acceso: 24 de septiembre de 2010]; 12: 273 - 287. URL disponible en: http://www.scielo.br/scielo.php?script = sci_arttext\&pid $=$ S141552731999000300007\&lng $=$ en

8. Axelsson P. Diagnosis and prediction of dental caries. New York: Quintenssence Publishing. p. 91 - 133.

9. Wanatabe $\mathrm{S}$, Ohnishi M, Imai K, Kawano E, Igarashi S. Estimation of the total saliva volume produced per day in five year old children. Arch Oral Biol 1995; 40: 781 - 782.

10. Kelsey JL, Douglas TH. Methods in Observational Epidemiology. New York: Oxford University Press, 1986. p. 244 $-257$.

11. Ibarra AM, Hernández D, Sampayo EP. Relación de caries de la infancia temprana con el flujo salival en escolares de 3 a 5 años del hogar infantil Bambi [Trabajo de Grado]. Bucaramanga: Universidad Santo Tomás; 2007.

12. CDC-OMS. Epi-Inf 6 Versión 6.04a Nov 1996

13. Vaisman B, Martínez MG. Asesoramiento dietético para el control de la caries en niños. Revista Latinoamericana de Ortodoncia y Odontopediatría [en línea] 11 páginas URL disponible en: http://www.ortodoncia.ws/publicaciones/2004/asesoramiento_dietetico_control_caries.asp

14. Pieschacon MP, Camargo DM. Acuerdo entre el índice de Green y Vermillion con el índice cuantificado de placa. Ponencia XI Encuentro Nacional de Investigación Odontológica. Universidad CES. Medellín; 2000.

15. Ojeda W, Sánchez J, Concha SC. Evaluación de la asociación entre capacidad fisica funcional y la higiene oral en los niños y las niñas que acuden a la consulta odontológi- cas a las clínicas integrales del niño de la Universidad Santo Tomás. Ustasalud 2007; 6: 29 - 36.

16. Bratall D, Hänsel-Petersson G. Cariogram - A multifactorial risk assessment model for a multifactorial disease. Community Dent Oral Epidemiol 2005; 33: 256 - 264.

17. Microsoft Excell 5.0, Microsoft corporation; 1997.

18. Stata Corp. Stata Statistical Software. Release 9.0 College station, T.X: Stata corporation; 2008.

19. Pagano M, Gauvreau K. Principles of Biostatistics. Belmont: Duxbury Press. p. 35 - 44, 257 - 263

20. Bautista LE. Métodos avanzados de análisis. Universidad Industrial de Santander. Bucaramanga, 2002.

21. Ministerio de Salud. Resolución No. 008430 del 4 de octubre de 1993.

22. Rethman J. Trends in preventive care: caries risk assessment and indications for sealants. J Am Dent Assoc 2000; 131: 7 - 12.

23. Sudha P, Bashin S, Anegund RT. Prevalence of dental caries among 5-13-year-old children of Mangore city. J Indian Soc Pedod Prev Dent 2005; 23: 74 - 79.

24. Burt BA, Pai S. Sugar consumption and caries risk: A systematic review. J Dent Educ 2001; 65: 1017 - 1023.

25. Reisine ST, Psoter W. Socieconomic status and selected behavioral determinants as risk factors for dental caries. J Dent Educ 2001; 65: 1009 - 1016.

26. Llena-Puy C. La saliva en el mantenimiento de la salud oral como ayuda en el diagnóstico de algunas patologías. Med Oral Patol Oral Cir Bucal 2006; 11: E449 - E455.

27. Mattos-Vela MA, Melgar-Hermoza RA. Riesgo de caries dental. Rev Estomatol Herediana 2004; 14: 101 - 106.

28. Kaplan I, Zuk-Paz L, Wolff A, Tel-Aviv PT, Harotzim I. Association between salivary flow rates, oral symptoms, and oral mucosal status. Oral Surg Oral Med Oral Pathol Oral Radiol Endod 2008; 106: 235 - 241.

29. Segovia-Villanueva A, Estrella-Rodríguez R, Medina-Solis $\mathrm{CE}$, Maupomé $\mathrm{G}$. Severidad de caries y factores asociados en preescolares de 3-6 años de edad en Campeche, México. Rev Salud Pública 2004; 7: 56 - 69.

30. Sánchez-Pérez TL, Sáenz-Martínez LP, Gómez-López ME, Pérez-Quiroz J. Resistencia del esmalte a la disolución ácida y su correlación con la caries dental. Salud Pública Méx 1995; 37: 224 - 231.

31. American Academy of Pediatric Dentistry. Policy on Early Childhood Caries (ECC): Unique challenges and treatment options. Reference Manual 2009; 31: 44 - 46.

32. Hernández-Ávila M, Garrido F, Salazar-Martínez E. Sesgo en estudios epidemiológicos. Salud Pública de México 2000; 42: 438 - 446.

\section{Correo electrónico de los autores:}

Sonia Patricia Suárez Muñoz: sopasumu@hotmail.com Mónica Tatiana Prieto Roa: monicatprieto@gmail.com Diana Carolina Otero Quintero: dicaotequi@hotmail.com Walter Alexander Ojeda Olarte: waltersan14@hotmail.com Sonia Constanza Concha Sánchez: sococosa@yahoo.com 